\title{
Switching Phase States of Chaotic Circuits Coupled by Time-Varying Resistor
}

\author{
Yoko Uwate and Yoshifumi Nishio \\ Dept. of Electrical and Electronics Engineering, Tokushima University \\ 2-1 Minami-Josanjima, Tokushima, Japan \\ Email: \{uwate,nishio\}@ee.tokushima-u.ac.jp
}

\begin{abstract}
In this study, two chaotic circuits coupled by a time-varying resistor are investigated. We assume that the timevarying resistor is realized by switching a positive and a negative resistors alternately. By carrying out circuit experiments and computer simulations, we confirm that interesting switching phase states between in-phase and anti-phase is observed.
\end{abstract}

\section{INTRODUCTION}

Synchronization phenomena in complex systems are very interesting to describe various higher-dimensional nonlinear phenomena in the field of natural science. Studies on synchronization phenomena of coupled oscillators are extensively carried out in various fields, physics [1]-[4], biology [5], [6] engineering [7]-[11] and so on. Because many researchers suggest that synchronization phenomena of coupled oscillators have some relations to information processing in the brain. We consider that it is very important to investigate the synchronization phenomena of coupled oscillators to realize a brain computer for the future engineering application.

On the other hand, there are some systems whose dissipation factors vary with time, for example, under the timevariation of the ambient temperature, an equation describing an object moving in a space with some friction and an equation governing a circuit with a resistor whose temperature coefficient is sensitive such as thermistor. However, there are few discussions about coupled oscillators coupling by a timevarying resistor.

In our previous research, we have investigated synchronization phenomena in van der Pol oscillators coupled by a time-varying resistor. We realized the time-varying resistor by switching a positive and a negative resistors periodically. By changing the duty ratio $p$, we confirmed that the characteristics of the synchronization phenomena changed as follows. First, for smaller $p$, the two coupled oscillators are synchronized only in anti-phase. Second, for intermediate $p$, the coexistence of the in-phase and the anti-phase synchronizations can be observed. Finally, for larger $p$, only the in-phase synchronization can be confirmed. We consider what complex synchronization phenomena can be observed, when chaotic circuits are coupled by time-varying resistor.

In this study, two chaotic circuits coupled by a time-varying resistor are investigated. First, the coexistence of in-phase and anti-phase synchronization are observed. Next, we confirm that interesting synchronization phenomena with switching phase states between the two chaotic circuits can be observed when the strength of coupling parameter $R$ is decreased.
Furthermore, the sojourn time of the in-phase and the antiphase are investigated. We confirm that the sojourn time depends on the frequency and the strength of the time-varying resistor.

\section{Circuit Model}

Figure 1 shows the circuit model, which is the chaotic version of the circuit investigated in [12]. In the circuit, two identical chaotic circuits are coupled by a time-varying resistor whose characteristics are shown in Fig. 2 [13].

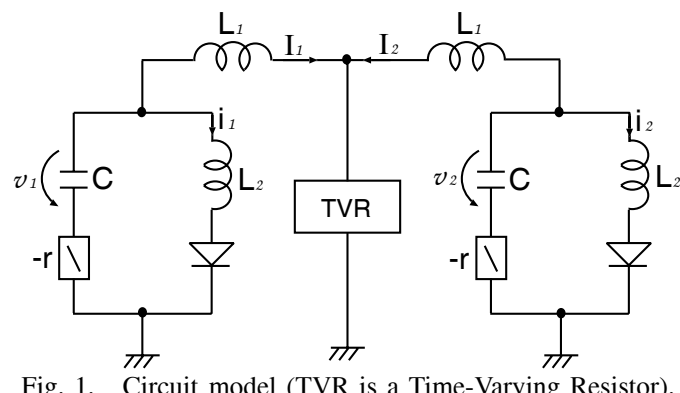

Fig. 1. Circuit model (TVR is a Time-Varying Resistor).

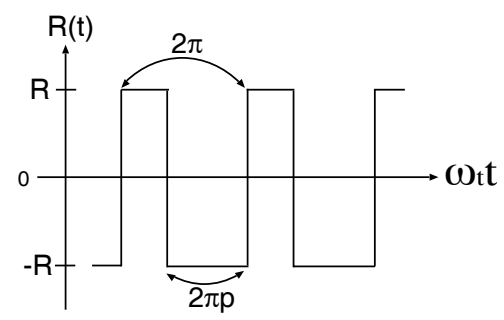

Fig. 2. Characteristics of the TVR.

First, the $i-v$ characteristics of the diodes are approximated by two-segment piecewise-linear functions as

$$
v_{d}\left(i_{k}\right)=0.5\left(r_{d} i_{k}+E-\left|r_{d} i_{k}-E\right|\right) .
$$

By changing the variables and parameters,

$$
\begin{gathered}
I_{k}=\sqrt{\frac{C}{L_{1}}} E x_{k}, \quad i_{k}=\sqrt{\frac{C}{L_{1}}} E y_{k}, \quad v_{k}=E z_{k}, \\
t=\sqrt{L_{1} C} \tau, \quad \alpha=\frac{L_{1}}{L_{2}}, \quad \beta=r \sqrt{\frac{C}{L_{1}}}, \\
\gamma=R \sqrt{\frac{C}{L_{1}}}, \quad \delta=r_{d} \sqrt{\frac{C}{L_{1}}}, \quad \omega=\frac{1}{\sqrt{L_{1} C}} \omega_{t},
\end{gathered}
$$


the normalized circuit equations are given as

$$
\left\{\begin{array}{l}
\frac{d x_{k}}{d \tau}=\beta\left(x_{k}+y_{k}\right)-z_{k} \pm \gamma\left(x_{1}+x_{2}\right) \\
\frac{d y_{k}}{d \tau}=\alpha \beta\left(x_{k}+y_{k}\right)-z_{k}-f\left(y_{k}\right) \\
\frac{d z_{k}}{d \tau}=x_{k}+y_{k} \quad(k=1,2)
\end{array}\right.
$$

where the sign of the coupling term changes according to the value of the time-varying resistor. The normalized characteristics of the diodes are given as

$$
f\left(y_{k}\right)=0.5\left(\delta y_{k}+1-\left|\delta y_{k}-1\right|\right) .
$$

\section{SyNCHRONIZATION PHENOMENA}

\section{A. In-Phase and Anti-Phase Synchronization}

We observed that the two coupled oscillators are synchronized in in-phase and anti-phase as shown in Figs. 3 and 4. These two synchronization states can be obtained by giving different initial conditions. The parameters of the chaotic circuits are fixed as $\alpha=7.0, \beta=0.084, \gamma=0.1$ and $\omega=1.924$.

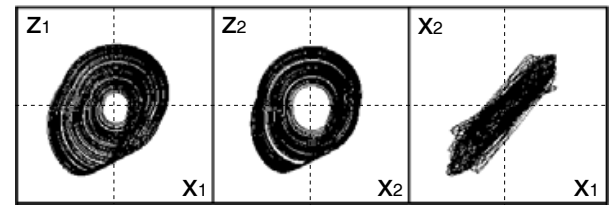

(a)

(b)

(c)

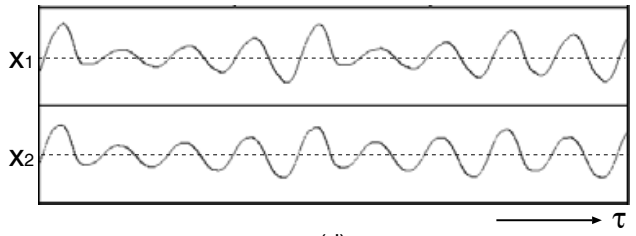

(d)

Fig. 3. In-phase synchronization (computer simulation results). (a) 1 st circuit attractor ( $x_{1}$ vs $z_{1}$ ). (b) 2 nd circuit attractor $\left(x_{2}\right.$ vs $\left.z_{2}\right)$. (c) Phase difference $\left(x_{1}\right.$ vs $\left.x_{2}\right)$. (d) Time wave form $\left(\tau\right.$ vs $x_{1}$ and $\left.x_{2}\right)$.

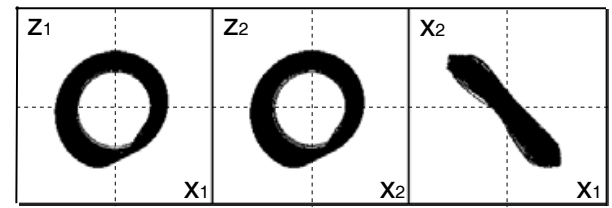

(a)

(b)

(c)

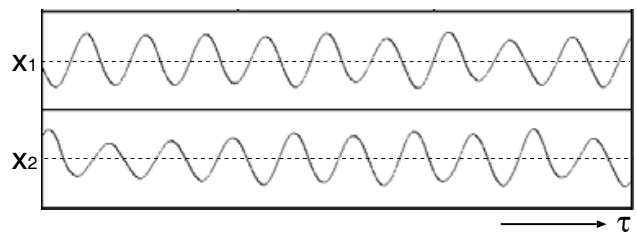

(d)

Fig. 4. Anti-phase synchronization (computer simulation results). (a) 1st circuit attractor $\left(x_{1}\right.$ vs $\left.z_{1}\right)$. (b) 2nd circuit attractor $\left(x_{2}\right.$ vs $\left.z_{2}\right)$. (c) Phase difference $\left(x_{1}\right.$ vs $\left.x_{2}\right)$. (d) Time wave form ( $\tau$ vs $x_{1}$ and $\left.x_{2}\right)$.

We also confirm that the two coupled chaotic circuits are synchronized in in-phase or at anti-phase in circuit experiments as shown in Figs. 5 and 6.

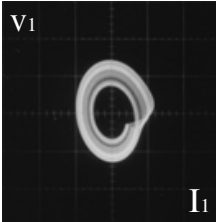

(a)

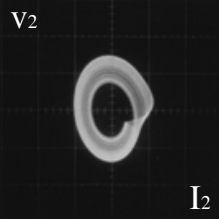

(b)

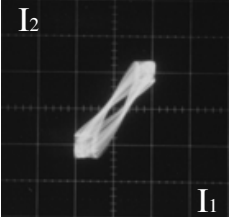

(c)

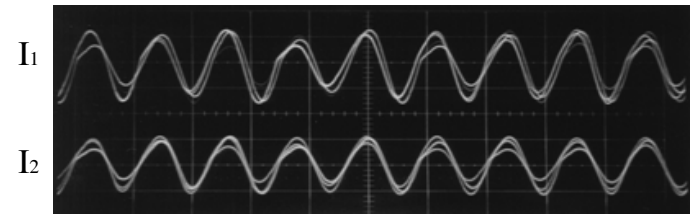

(d)

Fig. 5. In-phase synchronization (circuit experimental results). (a) 1st circuit Fig. 5. In-phase synchronization (circuit experimental results). (a) 1 st circuit
attractor $\left(I_{1}\right.$ vs $\left.v_{1}\right)$. (b) 2 nd circuit attractor $\left(I_{2}\right.$ vs $\left.v_{2}\right)$. (c) Phase difference attractor $\left(I_{1}\right.$ vs $\left.v_{1}\right)$. (b) 2 nd circuit attractor $\left(I_{2}\right.$ vs $\left.v_{2}\right)$. (c) Phase difference
$\left(I_{1}\right.$ vs $\left.I_{2}\right)$. (d) Time wave form $\left(t\right.$ vs $I_{1}$ and $\left.I_{2}\right) . L_{1}=300 \mathrm{mH}, L_{2}=$ $\left(I_{1}\right.$ vs $\left.I_{2}\right)$. (d) Time wave form $\left(t\right.$ vs $I_{1}$ and
$10 m H, C=33 n F, r=700 \Omega$ and $R=100 \Omega$.

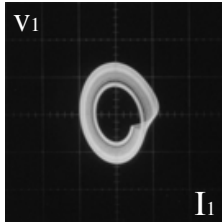

(a)

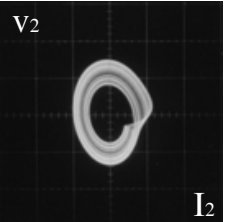

(b)

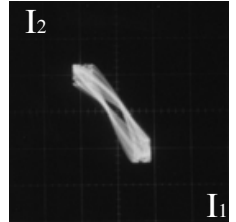

(c)

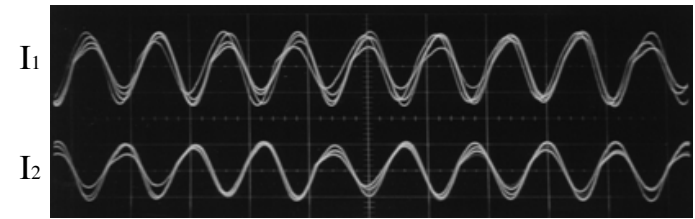

(d)

Fig. 6. Anti-phase synchronization (circuit experimental results). (a) 1st circuit attractor $\left(I_{1}\right.$ vs $\left.v_{1}\right)$. (b) 2 nd circuit attractor $\left(I_{2}\right.$ vs $\left.v_{2}\right)$. (c) Phase difference $\left(I_{1}\right.$ vs $\left.I_{2}\right)$. (d) Time wave form $\left(t\right.$ vs $I_{1}$ and $\left.I_{2}\right) . L_{1}=$ $300 \mathrm{mH}, L_{2}=10 \mathrm{mH}, \mathrm{C}=33 n F, r=700 \Omega$ and $R=100 \Omega$.

The one parameter bifurcation diagram of $x_{1}$ for in-phase and anti-phase synchronization modes are shown in Fig. 7. Figure 8 show the one parameter diagram of the phase difference. We can confirm the coexistence of in-phase and anti-phase synchronizations for $0.055<\beta<0.090$.

\section{B. Switching Phase States}

In this section, we investigate the synchronization phenomena when the strength of the coupling parameter $\gamma$ is decreased. We can confirm that the switching of the phase states between the in-phase and the anti-phase is observed as shown in Fig. 9. These switching phenomena could not be confirmed in the two van der Pol oscillators coupled by timevarying resistor. The chaotic circuits coupled by time-varying resistor has possibility to generate complex phenomena.

Next, we pay our attention to the sojourn time of the inphase state and the anti-phase state. We carry out the 30 moving average of the phase difference between two coupled chaotic circuits to distinguish the in-phase state and the antiphase state more correctly. The simulated result of the moving average of the phase difference is shown in Fig. 10. We 


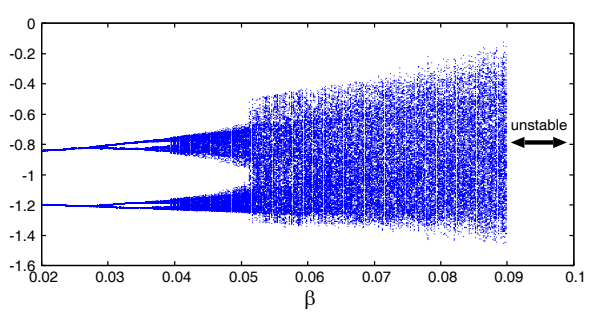

(a) Bifurcation diagram of $x_{1}$ for in-phase synchronization mode.

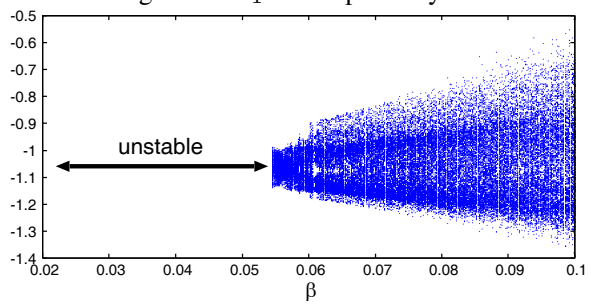

(b) Bifurcation diagram of $x_{1}$ for anti-phase synchronization mode Fig. 7. One parameter bifurcation diagrams for $\alpha=7.0, \gamma=0.1, \omega=$ 1.924. Horizontal axis: $\beta$.

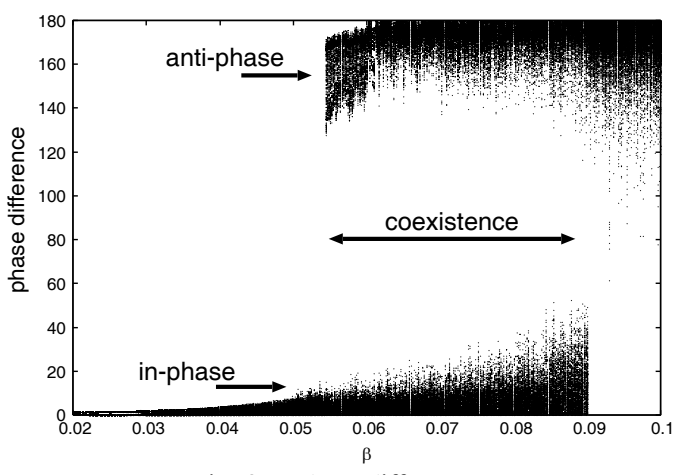

Fig. 8. Phase difference.

define in-phase or anti-phase synchronization, by using the 30 moving average of the phase difference. Namely, when the phase difference is smaller or larger than 90 degrees, the synchronization state of two chaotic circuits are determined to in-phase or anti-phase state.

The frequency distribution of the sojourn time of the synchronization states is investigated. Figure 11 shows the simulated results of the frequency distribution. From these figures, the frequency distribution of the in-phase and the antiphase is similar when the parameters of the chaotic circuits are set as follows: $\alpha=7.0, \beta=0.084, \gamma=0.095$ and $\omega=1.924$.

Furthermore, the average sojourn time of the in-phase state and the anti-phase state when the frequency $\omega$ of the timevarying resistor is changed. The simulated result is shown in Fig. 12. The horizontal axis is frequency $\omega$ and the vertical axis is average sojourn time. The average sojourn time of the in-phase state increase by increasing $\omega$. On the other hand, the average sojourn time of the anti-phase state is almost constant. From these results, we can see that the anti-phase state does not depends on the frequency $\omega$ of the time-varying resistor. When $\omega$ is smaller than 1.908 , two chaotic circuits coupled by time-varying resistor do not synchronous neither the in-phase state nor the anti-phase state. And $\omega$ is larger than 1.930 , the

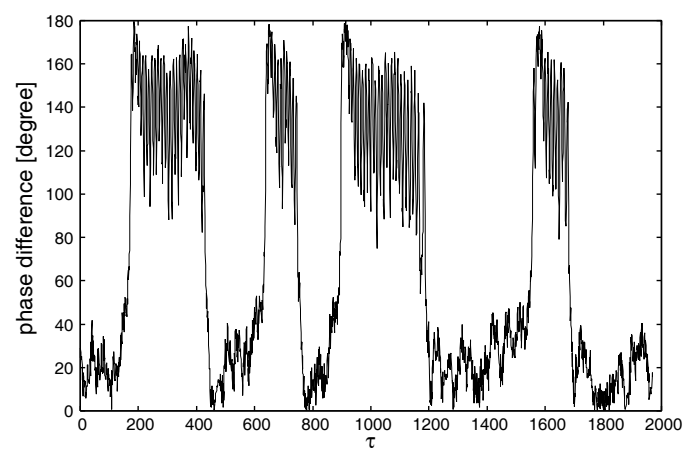

Fig. 9. Switching phase states $(\gamma=0.095)$.

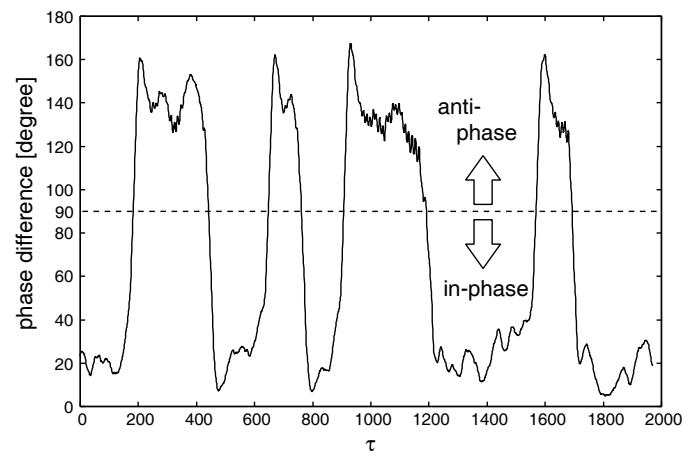

Fig. 10. Moving average of the phase states.

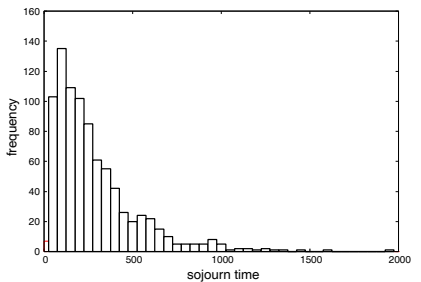

(a) In-phase.

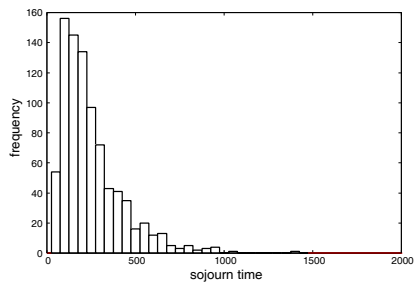

(b) Anti-phase.
Fig. 11. Frequency distribution of sojourn time.

only in-phase state can be occurred.

Some example of the switching synchronization state and the frequency distribution when $\omega$ are set to 1.922 and 1.926 are shown in Figs. 13 and 14, respectively. In the case of $\omega=1.922$, the sojourn time of the in-phase state is longer than the anti-phase state (Fig. 13). In the case of $\omega=1.926$, the sojourn time of the anti-phase state is longer than the inphase state (Fig. 14).

Finally, we investigate synchronization in dependence on the coupling strength $\gamma$. The average sojourn time of the in-phase state and the anti-phase state is shown in Fig. 15. The average sojourn time of the in-phase state increase by increasing $\gamma$. On the other hand, the average sojourn time of the anti-phase state is almost constant. From these results, we can see that the anti-phase state does not depends on $\gamma$ as well as frequency $\omega$.

\section{CONCLUSIONS}

In this study, we have investigated phase differences in two chaotic circuits coupled by a time-varying resistor when the 


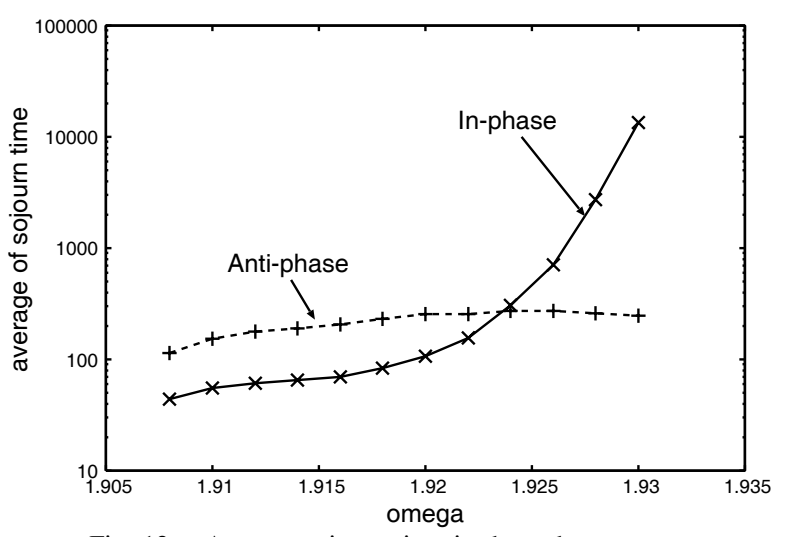

Fig. 12. Average sojourn time in dependence on $\omega$.

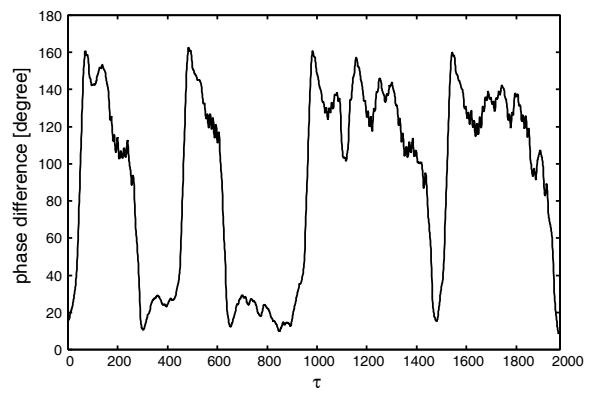

(a)

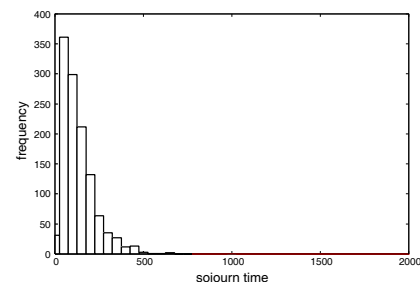

(b)

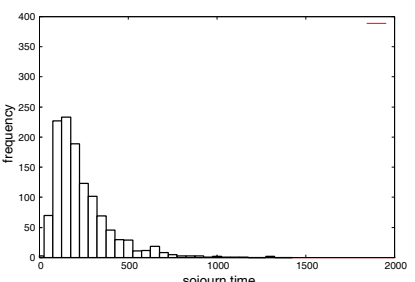

(c)
Fig. 13. Switching phase state for $\omega=1.922$. (a) Moving average of switching synchronization state. (b) Frequency distribution of sojourn time (in-phase). (c) Frequency distribution of sojourn time (in-phase).

frequency of the time-varying resistor is changed. By carrying out computer calculations, we can confirmed that the synchronization phenomena of the two coupled chaotic circuits is depend on the frequency of the time-varying resistor. Further, we investigated the sojourn time of the synchronization state when the frequency $\omega$ and coupling strength $\gamma$ are changed. The average sojourn time of the anti-phase has no influence for changing $\omega$ and $\gamma$.

\section{REFERENCES}

[1] L.L. Bonilla, C.J. Perez Vicente and R. Spigler, "Time-periodic phases in populations of nonlinearly coupled oscillators with bimodal frequency distributions," Physica D: Nonlinear Phenomena, vol.113, no.1, pp.79-97, Feb. 1998.

[2] J.A. Sherratt, "Invading wave fronts and their oscillatory wakes are linked by a modulated traveling phase resetting wave," Physica D: Nonlinear Phenomena, vol.117, no.1-4, pp.145-166, June 1998.

[3] G. Abramson, V.M. Kenkre and A.R. Bishop, "Analytic solutions for nonlinear waves in coupled reacting systems," Physica A: Statistical Mechanics and its Applications, vol.305, no.3-4, pp.427-436, Mar. 2002.

[4] I. Belykh, M. Hasler, M. Lauret and H. Nijmeijer, "Synchronization and graph topology," Int. J. Bifurcation and Chaos, vol.15, no.11, pp.34233433, Nov. 2005

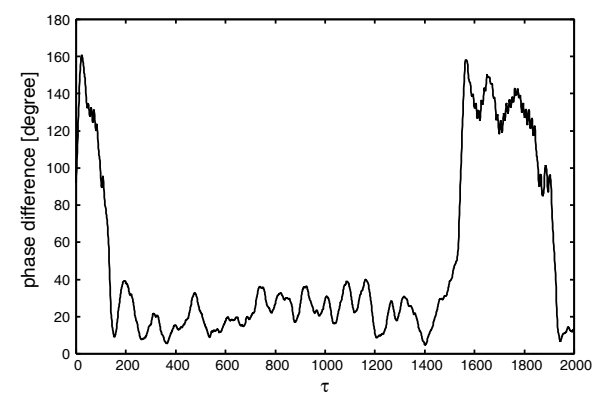

(a) Moving average of switching synchronization state.

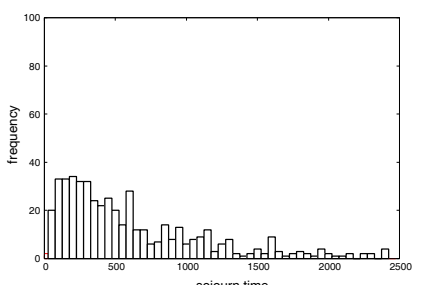

(b)

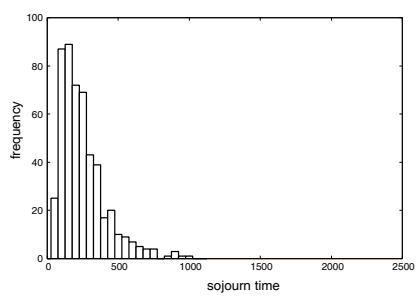

(c)
Fig. 14. Switching phase state for $\omega=1.926$. (a) Moving average of switching synchronization state. (b) Frequency distribution of sojourn time (in-phase). (c) Frequency distribution of sojourn time (in-phase).

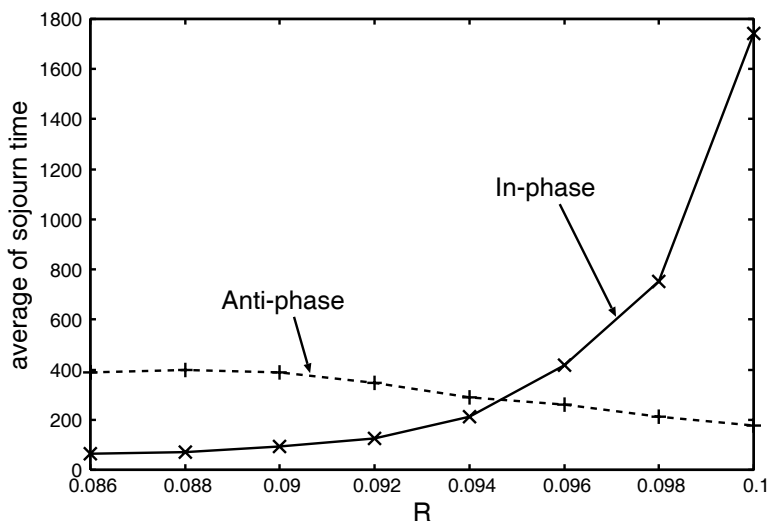

Fig. 15. Average sojourn time in dependence on $\gamma$.

[5] C.M. Gray, "Synchronous oscillations in neural systems: mechanisms and functions," J. Computational Neuroscience, vol.1, pp.11-38, 1994.

[6] J. Cosp, J. Madrenas, E. Alarcon, E. Vidal and G. Villar, "Synchronization of nonlinear electronic oscillators for neural computation," IEEE Trans. Neural Networks, vol.15, no.5, pp.1315-1327, Sep. 2004.

[7] T. Suezaki and S. Mori, "Mutual synchronization of two oscillators," Trans. IECE, vol.48, no.9, pp.1551-1557, Sep. 1965.

[8] S.P. Datardina and D.A. Linkens, "Multimode oscillations in mutually coupled van der Pol type oscillators with fifth-power nonlinear characteristics," IEEE Trans. Circuits Syst., vol.CAS-25, no.5, pp.308-315, May 1978.

[9] Y. Nishio and S. Mori, "Mutually coupled oscillators with an extremely large number of steady states," Proc. of ISCAS'92, vol.2, pp.819-822, May 1992.

[10] M. Yamauchi, M. Wada, Y. Nishio and A. Ushida, "Wave propagation phenomena of phase states in oscillators coupled by inductors as a ladder," IEICE Trans. Fundamentals, vol.E82-A, no.11, pp.2592-2598, Nov. 1999.

[11] B. Liu, X. Liu, G. Chen and H. Wang, "Robust impulsive synchronization of uncertain dynamical networks," IEEE Trans. Circuits Syst. I, vol.52, no.7, pp.1431-1441, July 2005.

[12] Y. Uwate and Y. Nishio, "Synchronization phenomena in van der Pol oscillators coupled by a time-varying resistor," International Journal of Bifurcation and Chaos (to appear).

[13] Y. Nishio and S. Mori, "Chaotic phenomena in nonlinear circuits with time-varying resistors," IEICE Trans. Fundamentals, vol.E76-A, no.3, pp.467-475, Mar. 1993. 